CRYSTALLOGRAPHIC COMMUNICATIONS

ISSN 2056-9890

Received 20 September 2014

Accepted 26 February 2015

Edited by W. T. A. Harrison, University of Aberdeen, Scotland

Keywords: crystal structure; aldol; methylation; acetylanthracene; stereochemistry

CCDC reference: 1051418

Supporting information: this article has supporting information at journals.iucr.org/e
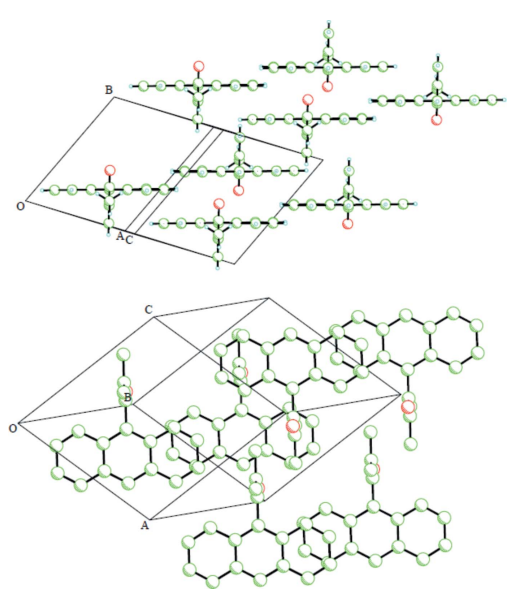

OPEN $\odot$ ACCESS

\section{Crystal structure of 9-methacryloylanthracene}

\author{
Aditya Agrahari, ${ }^{\text {a }}$ Patrick O. Wagers, ${ }^{\mathrm{b}}$ Steven M. Schildcrout, ${ }^{\mathrm{c}}$ John Masnovi ${ }^{\mathrm{a} *}$ and \\ Wiley J. Youngs ${ }^{\mathrm{b}}$
}

${ }^{\mathbf{a}}$ Department of Chemistry, Cleveland State University, Cleveland $\mathrm{OH} 44115$, USA, ${ }^{\mathbf{b}}$ Department of Chemistry, University
of Akron, Akron OH 44325, USA, and ${ }^{\mathbf{c}}$ Department of Chemistry, Youngstown State University, Youngstown OH 44555 ,
USA. *Correspondence e-mail: j.masnovi@csuohio.edu

In the title compound, $\mathrm{C}_{18} \mathrm{H}_{14} \mathrm{O}$, with systematic name 1-(anthracen-9-yl)-2methylprop-2-en-1-one, the ketonic $\mathrm{C}$ atom lies 0.2030 (16) $\AA$ out of the anthrylring-system plane. The dihedral angle between the planes of the anthryl and methacryloyl moieties is $88.30(3)^{\circ}$ and the stereochemistry about the $\mathrm{Csp} p^{2}-$ $\mathrm{Cs} p^{2}$ bond in the side chain is transoid. In the crystal, the end rings of the anthryl units in adjacent molecules associate in parallel-planar orientations [shortest centroid-centroid distance $=3.6320$ (7) $\AA$ ] . A weak hydrogen bond is observed between an aromatic $\mathrm{H}$ atom and the $\mathrm{O}$ atom of a molecule displaced by translation in the $a$-axis direction, forming sheets of parallel-planar anthryl groups packing in this direction.

\section{Chemical Context}

Enolizable aldehydes react with formaldehyde in strong aqueous base to form polyols, whereas ketones usually react to form polyhydroxyketones (Davidson \& Bogert, 1935; Vik et al., 1973; Weissermel \& Arpe, 1997; Wittcoff et al., 2013). Therefore, the observed methylation of 9-acetylanthracene by formaldehyde with alcoholic potassium carbonate (see Scheme below) is remarkable in that the reaction occurs with weak base in a non-aqueous medium by reduction of formaldehyde to form the methyl group (Pande et al., 1998). Consequently, we obtained an X-ray structure determination to confirm the identity of the isolated product, 9-methacryloylanthracene or 1-(9-anthryl)-2-methyl-2-propen-1-one.<smiles>CC(=O)c1c2ccccc2cc2ccccc12</smiles><smiles>O=C[18F]</smiles><smiles>CCCCC(C)O</smiles><smiles>C=C(C)C(=O)c1c2ccccc2cc2ccccc12</smiles>

\section{Structural commentary}

The crystal structure (Fig. 1) establishes the material to be the $\alpha$-methylated aldol condensation product. Bond distances and valence angles agree well between the observed and the calculated structures. The anthryl ring system is essentially planar, as is the methacryloyl substituent (excepting the hydrogen atoms of the methyl group), whereas the calculated structure shows a slight deviation, about $7^{\circ}$, of the methacryloyl skeleton from planarity. The substituted $\mathrm{C}$ atom (C9) of the anthryl group also lies in the plane of the substituent, deviating by only 0.002 (2) ^. However, this C atom is puckered, so that the carbonyl $\mathrm{C}$ atom resides 0.2030 (16) $\AA$ out of the anthryl plane. This puckering is 


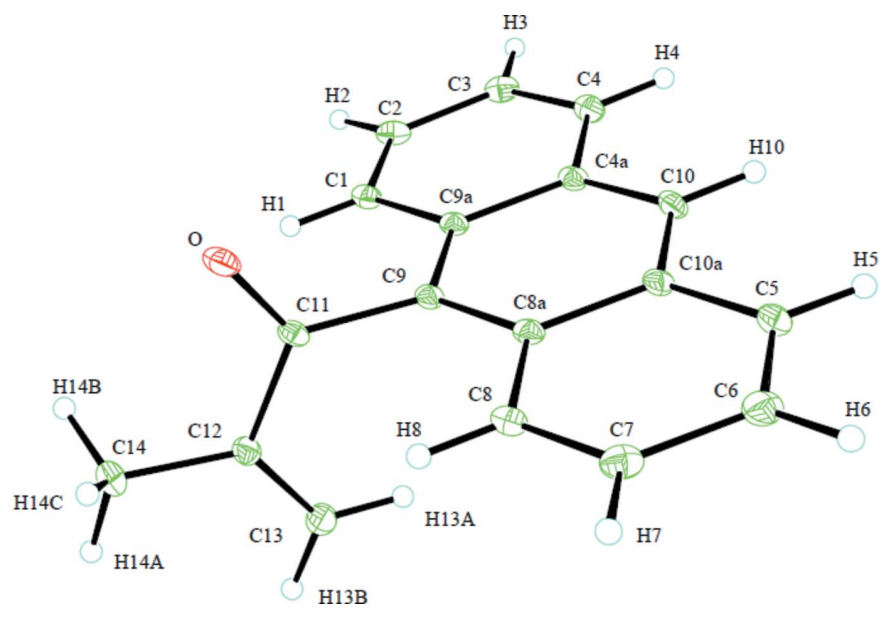

Figure 1

ORTEP (30\% probability elipsoids) plot of the title compound showing the atom-labeling scheme.

absent in the calculated structure. The planes of the anthryl and methacryloyl moieties are nearly perpendicular with a dihedral angle of $88.30(3)^{\circ}$ (but about $12^{\circ}$ from perpendicular in the calculated structure). This general orientation is demanded by the close intramolecular approach of the methacryloyl group to the peri- $\mathrm{H}$ atoms ( $\mathrm{H} 1$ and $\mathrm{H} 8)$, but packing effects may also contribute to deciding the exact angle since that calculated for the energy minimum differs by about $10^{\circ}$ from that observed. The observed positioning is not quite
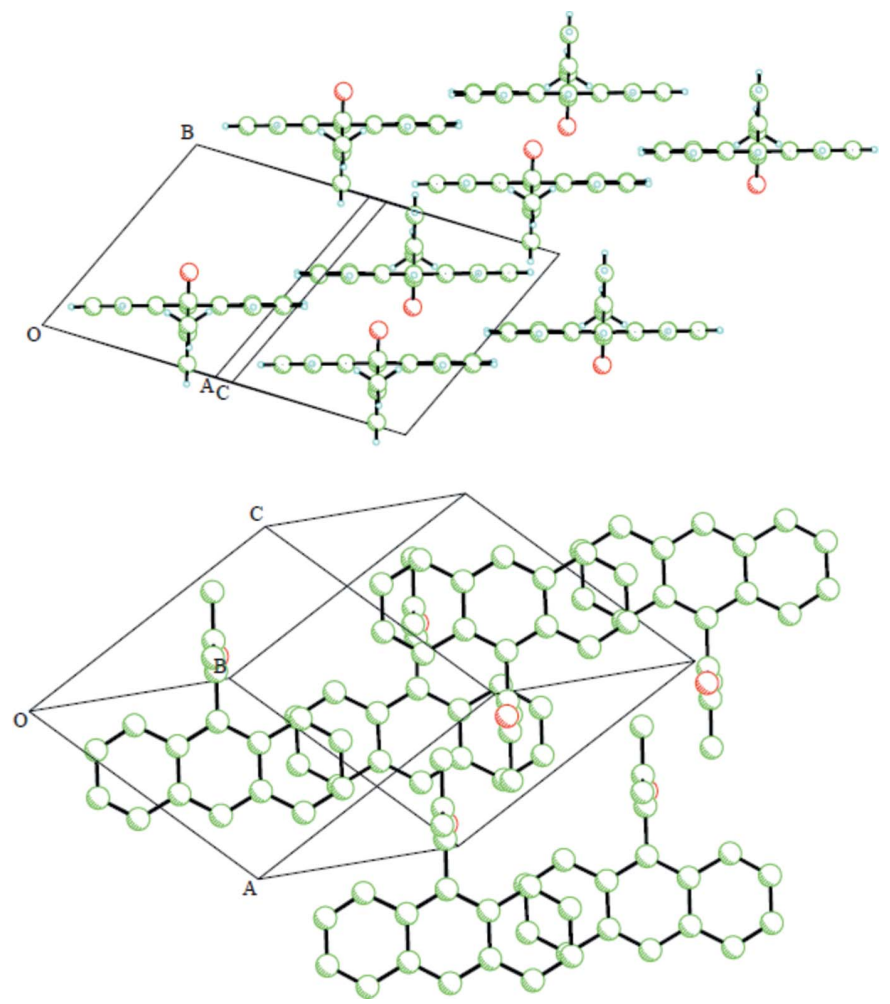

Figure 2

Views parallel to the planes of both the anthryl and the methacryloyl moieties (top) and parallel to the methacryloyl but perpendicular to the anthryl with $\mathrm{H}$ atoms omitted for clarity (bottom).
Table 1

Hydrogen-bond geometry $\left(\AA{ }^{\circ}\right)$.

\begin{tabular}{lllll}
\hline$D-\mathrm{H} \cdots A$ & $D-\mathrm{H}$ & $\mathrm{H} \cdots A$ & $D \cdots A$ & $D-\mathrm{H} \cdots A$ \\
\hline $\mathrm{C} 3-\mathrm{H} 3 \cdots \mathrm{O}^{\mathrm{i}}$ & 0.95 & 2.48 & $3.3747(16)$ & 157 \\
\hline
\end{tabular}

Symmetry code: (i) $x-1, y, z$.

symmetrical, with $\mathrm{C} 11$ being slightly closer $(0.018 \AA)$ to $\mathrm{H} 1$ than to H8. Similar geometries are found in 9-acetylanthracene, with a dihedral angle of 88.70 (3) (Andersson et al., 1984) and in 9-(bromoacetyl)anthracene, with a dihedral angle of $74.2(1)^{\circ}$ (Kubo et al., 2007). Unfavorable non-bonded interactions in the present structure are likely the reason that the methyl group, which is bulkier than the methylene group, projects away from the anthryl moiety, making the stereochemistry of the $\mathrm{C} 11-\mathrm{C} 12$ bond transoid. The puckering observed at $\mathrm{C} 9$ would partially relieve these unfavorable steric interactions occurring about this position.

\section{Supramolecular features}

Intermolecular close contacts between large aromatic groups in the solid state often involve $\pi-\pi$ stacking interactions involving parallel planar associations (Główka et al., 1999). This motif is observed here as well, with the anthryl rings displaced and stacking alternately with those of neighboring molecules (Fig. 2). The centroid-centroid separations are 3.6320 (7) and 3.7532 (7) and 3.7807 (8) ̊. The methacryloyl substituent prevents such interactions involving the central ring of the anthryl moiety. A weak hydrogen bond is observed (Fig. 3) between an aromatic $\mathrm{H}$ atom (H3) and the $\mathrm{O}$ atom of a molecule displaced by translation in the $a$-axis direction

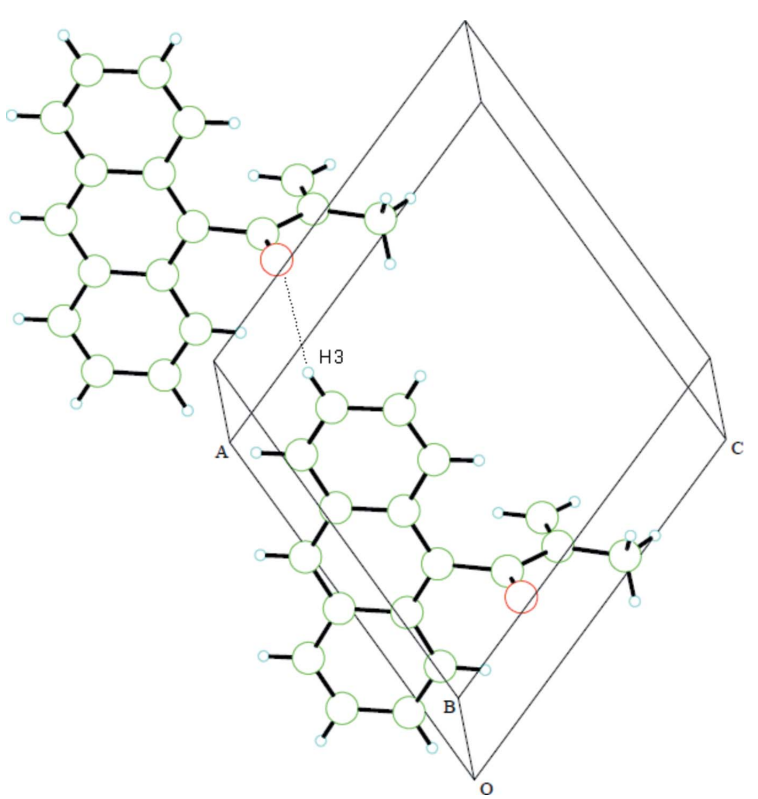

Figure 3

A fragment of a [100] hydrogen-bonded chain of molecules in the crystal showing the intermolecular $\mathrm{O} \cdots \mathrm{H}$ close contact (dotted line). 
(Table 1), resulting in the formation of anthryl groups packing in parallel-planar sheets in this direction.

\section{Synthesis and crystallization}

Refluxing 9-acetylanthracene (1.0 g), paraformaldehyde $(273 \mathrm{mg})$, and potassium carbonate $(942 \mathrm{mg})$ in $3.0 \mathrm{ml}$ ethanol afforded $80 \mathrm{mg}$ product which eluted first from an alumina column with $10 \%$ ethyl acetate-hexane and was crystallized from chloroform-hexane in the form of colorless plates.

\section{Refinement}

Crystal data, data collection and structure refinement details are summarized in Table 2. The $\mathrm{H}$ atoms were placed in calculated positions and refined as riding atoms, with $\mathrm{C}-\mathrm{H}=$ $0.95 \AA$ and $U_{\text {iso }}(\mathrm{H})=1.2 U_{\text {eq }}(\mathrm{C}$-alkene and C-aromatic $)$, and $\mathrm{C}-\mathrm{H}=0.98 \AA$ and $U_{\text {iso }}(\mathrm{H})=1.5 U_{\text {eq }}(\mathrm{C}$-methyl $)$.

\section{Calculations}

Density-functional theoretical computations were performed using Gaussian software (Frisch et al., 2010) through the Ohio Supercomputing Center (in Columbus $\mathrm{OH}$ ) with Zhao and Truhlar's hybrid meta exchange-correlation functional, M062X, (Choe, 2012; Huh \& Choe, 2010; Zhao \& Truhlar, 2008), which is parameterized for non-metallic systems with noncovalent $\pi-\pi$ interactions for accurate modelling of intramolecular dispersion effects. The basis set used is $6-31+G(d)$. To obtain the geometry at the global minimum potential energy, optimization was based on the minimum-energy conformation from a two-torsion MM2 plot (ChemBio3D Ultra 12.0; www.CambridgeSoft.com) using rotations about the $\mathrm{C} 9-\mathrm{C} 11$ and $\mathrm{C} 11-\mathrm{C} 12$ single bonds. The M06-2X structure has all vibrational frequencies positive, verifying that it is at a potential-energy minimum. Calculated values for geometrical paramters in the optimized isolated molecule are given in the Supporting information.

\section{Acknowledgements}

The authors would like to thank the Graduate College and Chemistry Department at Cleveland State University for support, the Ohio Supercomputing Center for a grant of computer time, and the National Science Foundation (CHE0840446) for funds used to purchase the Bruker APEXII DUO X-ray diffractometer used in this research.
Table 2

Experimental details.

\begin{tabular}{|c|c|}
\hline \multicolumn{2}{|l|}{ Crystal data } \\
\hline Chemical formula & $\mathrm{C}_{18} \mathrm{H}_{14} \mathrm{O}$ \\
\hline$M_{\mathrm{r}}$ & 246.29 \\
\hline Crystal system, space group & Triclinic, $P \overline{1}$ \\
\hline Temperature $(\mathrm{K})$ & 100 \\
\hline$a, b, c(\AA)$ & $8.7602(5), 9.1784(5), 9.2032(5)$ \\
\hline$\alpha, \beta, \gamma\left({ }^{\circ}\right)$ & $67.206(2), 71.670(3), 75.195(2)$ \\
\hline$V\left(\AA^{3}\right)$ & $639.98(6)$ \\
\hline$Z$ & 2 \\
\hline Radiation type & Mo $K \alpha$ \\
\hline$\mu\left(\mathrm{mm}^{-1}\right)$ & 0.08 \\
\hline Crystal size $(\mathrm{mm})$ & $0.21 \times 0.17 \times 0.05$ \\
\hline \multicolumn{2}{|l|}{ Data collection } \\
\hline Diffractometer & Bruker APEXII CCD \\
\hline Absorption correction & $\begin{array}{l}\text { Multi-scan (SADABS; Bruker, } \\
\text { 1997) }\end{array}$ \\
\hline$T_{\min }, T_{\max }$ & $0.984,0.996$ \\
\hline $\begin{array}{l}\text { No. of measured, independent and } \\
\text { observed }[I>2 \sigma(I)] \text { reflections }\end{array}$ & $12757,2590,2348$ \\
\hline$R_{\text {int }}$ & 0.026 \\
\hline$(\sin \theta / \lambda)_{\max }\left(\AA^{-1}\right)$ & 0.623 \\
\hline \multicolumn{2}{|l|}{ Refinement } \\
\hline$R\left[F^{2}>2 \sigma\left(F^{2}\right)\right], w R\left(F^{2}\right), S$ & $0.036,0.106,1.06$ \\
\hline No. of reflections & 2590 \\
\hline No. of parameters & 173 \\
\hline $\mathrm{H}$-atom treatment & H-atom parameters constrained \\
\hline$\Delta \rho_{\max }, \Delta \rho_{\min }\left(\mathrm{e} \AA^{-3}\right)$ & $0.26,-0.21$ \\
\hline
\end{tabular}

Computer programs: APEX2 and SAINT (Bruker, 1997), SHELXS97 and SHELXTL (Sheldrick, 2008).

\section{References}

Andersson, K., Becker, H. D., Engelhardt, L. M., Hansen, L. \& White, A. H. (1984). Aust. J. Chem. 37, 1337-1340.

Bruker (1997). APEX2, SAINT and SADABS. Bruker AXS Inc., Madison, Wisconsin, USA.

Choe, S. J. (2012). Bull. Korean Chem. Soc. 33, 2861-2866.

Davidson, D. \& Bogert, M. T. (1935). J. Am. Chem. Soc. 57, 905-905.

Frisch, M. J. et al. (2010). Gaussian 09. Revision C.01. Gaussian Inc., Wallingford, CT, USA.

Główka, M. L., Martynowski, D. \& Kozłowska, K. (1999). J. Mol. Struct. 474, 81-89.

Huh, D. S. \& Choe, S. J. (2010). J. Porphyrins Phthalocyanines, 14, 592-604.

Kubo, K., Watanabe, K. \& Sakurai, T. (2007). Acta Cryst. E63, o1300o1301.

Pande, P. P., Joshi, G. C. \& Mathela, C. S. (1998). Synth. Commun. 28, 4193-4200.

Sheldrick, G. M. (2008). Acta Cryst. A64, 112-122.

Vik, J. E., Kierkegaard, C., Pappas, J., Skaarup, S., Aaltonen, R. \& Swahn, C. G. (1973). Acta Chem. Scand. 27, 251-257.

Weissermel, K. \& Arpe, H.-J. (1997). In Industrial Organic Chemistry. Weinheim: VCH Verlagsgeselshaft.

Wittcoff, H. A., Reuben, B. G. \& Plotkin, J. S. (2013). In Industrial Organic Chemicals, 2nd ed. Hoboken, NJ: Wiley.

Zhao, Y. \& Truhlar, D. G. (2008). Theor. Chem. Acc. 120, 215-241. 


\section{supporting information}

Acta Cryst. (2015). E71, 357-359 [doi:10.1107/S2056989015004090]

\section{Crystal structure of 9-methacryloylanthracene}

Aditya Agrahari, Patrick O. Wagers, Steven M. Schildcrout, John Masnovi and Wiley J. Youngs

Computing details

Data collection: APEX2 (Bruker, 1997); cell refinement: SAINT (Bruker, 1997); data reduction: SAINT (Bruker, 1997); program(s) used to solve structure: SHELXS97 (Sheldrick, 2008); program(s) used to refine structure: SHELXS97

(Sheldrick, 2008); molecular graphics: SHELXTL (Sheldrick, 2008); software used to prepare material for publication: SHELXTL (Sheldrick, 2008).

1-(Anthracen-9-yl)-2-methylprop-2-en-1-one

Crystal data

$\mathrm{C}_{18} \mathrm{H}_{14} \mathrm{O}$

$M_{r}=246.29$

Triclinic, $P \overline{1}$

Hall symbol: -P 1

$a=8.7602(5) \AA$

$b=9.1784(5) \AA$

$c=9.2032(5) \AA$

$\alpha=67.206(2)^{\circ}$

$\beta=71.670(3)^{\circ}$

$\gamma=75.195(2)^{\circ}$

$V=639.98(6) \AA^{3}$

$Z=2$

$F(000)=260$

$D_{\mathrm{x}}=1.278 \mathrm{Mg} \mathrm{m}^{-3}$

Mo $K \alpha$ radiation, $\lambda=0.71073 \AA$

Cell parameters from 9953 reflections

$\theta=2.5-28.4^{\circ}$

$\mu=0.08 \mathrm{~mm}^{-1}$

$T=100 \mathrm{~K}$

Plate, colourless

$0.21 \times 0.17 \times 0.05 \mathrm{~mm}$

Data collection

Bruker APEXII CCD

diffractometer

Radiation source: fine-focus sealed tube

Graphite monochromator

$\varphi$ and $\omega$ scans

Absorption correction: multi-scan

(SADABS; Bruker, 1997)

$T_{\min }=0.984, T_{\max }=0.996$

12757 measured reflections

2590 independent reflections

2348 reflections with $I>2 \sigma(I)$

$R_{\text {int }}=0.026$

$\theta_{\text {max }}=26.3^{\circ}, \theta_{\min }=2.4^{\circ}$

$h=-10 \rightarrow 10$

$k=-11 \rightarrow 11$

$l=-11 \rightarrow 11$

Refinement

Refinement on $F^{2}$

Least-squares matrix: full

$R\left[F^{2}>2 \sigma\left(F^{2}\right)\right]=0.036$

$w R\left(F^{2}\right)=0.106$

$S=1.06$

2590 reflections

173 parameters

0 restraints

Primary atom site location: structure-invariant

direct methods

Secondary atom site location: difference Fourier map

Hydrogen site location: inferred from

neighbouring sites

$\mathrm{H}$-atom parameters constrained

$w=1 /\left[\sigma^{2}\left(F_{\mathrm{o}}^{2}\right)+(0.0479 P)^{2}+0.2652 P\right]$

where $P=\left(F_{\mathrm{o}}^{2}+2 F_{\mathrm{c}}{ }^{2}\right) / 3$

$(\Delta / \sigma)_{\max }<0.001$

$\Delta \rho_{\max }=0.26{\mathrm{e} \AA^{-3}}^{-3}$

$\Delta \rho_{\min }=-0.21 \mathrm{e} \AA^{-3}$ 


\section{Special details}

Geometry. All e.s.d.'s (except the e.s.d. in the dihedral angle between two l.s. planes) are estimated using the full covariance matrix. The cell e.s.d.'s are taken into account individually in the estimation of e.s.d.'s in distances, angles and torsion angles; correlations between e.s.d.'s in cell parameters are only used when they are defined by crystal symmetry. An approximate (isotropic) treatment of cell e.s.d.'s is used for estimating e.s.d.'s involving l.s. planes.

Refinement. Refinement of $F^{2}$ against ALL reflections. The weighted $R$-factor $w R$ and goodness of fit $S$ are based on $F^{2}$, conventional $R$-factors $R$ are based on $F$, with $F$ set to zero for negative $F^{2}$. The threshold expression of $F^{2}>\sigma\left(F^{2}\right)$ is used only for calculating $R$-factors (gt) etc. and is not relevant to the choice of reflections for refinement. $R$-factors based on $F^{2}$ are statistically about twice as large as those based on $F$, and $R$ - factors based on ALL data will be even larger.

Fractional atomic coordinates and isotropic or equivalent isotropic displacement parameters $\left(\AA^{2}\right)$

\begin{tabular}{|c|c|c|c|c|}
\hline & $x$ & $y$ & $z$ & $U_{\text {iso }} * / U_{\text {eq }}$ \\
\hline $\mathrm{O}$ & $0.88771(10)$ & $0.57102(9)$ & $0.67719(10)$ & $0.0221(2)$ \\
\hline $\mathrm{C} 1$ & $0.49492(14)$ & $0.63847(13)$ & $0.64670(14)$ & $0.0176(2)$ \\
\hline $\mathrm{C} 2$ & $0.35652(15)$ & $0.59642(13)$ & $0.64748(15)$ & $0.0203(3)$ \\
\hline $\mathrm{C} 3$ & $0.21748(14)$ & $0.59128(14)$ & $0.77952(15)$ & $0.0214(3)$ \\
\hline $\mathrm{C} 4$ & $0.22034(14)$ & $0.63126(13)$ & $0.90640(14)$ & $0.0202(3)$ \\
\hline $\mathrm{C} 4 \mathrm{a}$ & $0.36214(13)$ & $0.67831(13)$ & $0.91022(14)$ & $0.0164(2)$ \\
\hline $\mathrm{C} 5$ & $0.51116(15)$ & $0.80977(14)$ & $1.17279(14)$ & $0.0206(3)$ \\
\hline C6 & 0.64792 (16) & $0.85245(14)$ & $1.17433(14)$ & $0.0229(3)$ \\
\hline $\mathrm{C} 7$ & $0.78992(15)$ & $0.85161(14)$ & $1.04616(15)$ & $0.0223(3)$ \\
\hline $\mathrm{C} 8$ & $0.79096(14)$ & $0.80876(13)$ & $0.91974(14)$ & $0.0183(3)$ \\
\hline $\mathrm{C} 8 \mathrm{a}$ & $0.64945(13)$ & $0.76406(12)$ & $0.91228(13)$ & $0.0153(2)$ \\
\hline $\mathrm{C} 9$ & $0.64571(13)$ & $0.71974(12)$ & $0.78388(13)$ & $0.0148(2)$ \\
\hline $\mathrm{C} 9 \mathrm{a}$ & $0.50386(13)$ & $0.67960(12)$ & $0.77832(13)$ & $0.0152(2)$ \\
\hline $\mathrm{C} 10$ & $0.36743(14)$ & $0.71921(13)$ & $1.03939(13)$ & $0.0179(3)$ \\
\hline C10a & $0.50634(14)$ & $0.76402(13)$ & $1.04251(13)$ & $0.0166(2)$ \\
\hline C11 & $0.80055(13)$ & $0.70042(13)$ & $0.65638(13)$ & $0.0150(2)$ \\
\hline $\mathrm{C} 12$ & $0.84479(14)$ & $0.83810(13)$ & $0.50679(13)$ & $0.0178(2)$ \\
\hline $\mathrm{C} 13$ & $0.74810(16)$ & $0.97827(14)$ & $0.48462(15)$ & $0.0242(3)$ \\
\hline $\mathrm{C} 14$ & $1.00033(15)$ & $0.80523(15)$ & $0.38767(15)$ & $0.0264(3)$ \\
\hline H1 & 0.5869 & 0.6405 & 0.5575 & $0.021 *$ \\
\hline $\mathrm{H} 2$ & 0.3528 & 0.5701 & 0.5586 & $0.024 *$ \\
\hline H3 & 0.1223 & 0.5599 & 0.7793 & $0.026^{*}$ \\
\hline $\mathrm{H} 4$ & 0.1265 & 0.6278 & 0.9939 & $0.024 *$ \\
\hline H5 & 0.4172 & 0.8103 & 1.2596 & $0.025^{*}$ \\
\hline H6 & 0.6486 & 0.8830 & 1.2616 & $0.027^{*}$ \\
\hline $\mathrm{H} 7$ & 0.8851 & 0.8812 & 1.0487 & $0.027^{*}$ \\
\hline H8 & 0.8871 & 0.8086 & 0.8353 & $0.022 *$ \\
\hline $\mathrm{H} 10$ & 0.2741 & 0.7164 & 1.1273 & $0.022 *$ \\
\hline H13A & 0.6505 & 0.9906 & 0.5644 & $0.029 *$ \\
\hline H13B & 0.7765 & 1.0662 & 0.3890 & $0.029 *$ \\
\hline H14A & 1.0155 & 0.9009 & 0.2910 & $0.040 *$ \\
\hline H14B & 0.9950 & 0.7164 & 0.3564 & $0.040^{*}$ \\
\hline $\mathrm{H} 14 \mathrm{C}$ & 1.0920 & 0.7770 & 0.4379 & $0.040 *$ \\
\hline
\end{tabular}


Atomic displacement parameters $\left(\AA^{2}\right)$

\begin{tabular}{lllllll}
\hline & $U^{11}$ & $U^{22}$ & $U^{33}$ & $U^{12}$ & $U^{13}$ & $U^{23}$ \\
\hline O & $0.0204(4)$ & $0.0189(4)$ & $0.0224(4)$ & $0.0011(3)$ & $-0.0021(3)$ & $-0.0070(3)$ \\
C1 & $0.0187(5)$ & $0.0149(5)$ & $0.0175(5)$ & $-0.0013(4)$ & $-0.0045(4)$ & $-0.0042(4)$ \\
C2 & $0.0244(6)$ & $0.0157(5)$ & $0.0228(6)$ & $-0.0011(4)$ & $-0.0109(5)$ & $-0.0057(4)$ \\
C3 & $0.0177(6)$ & $0.0166(5)$ & $0.0285(6)$ & $-0.0039(4)$ & $-0.0105(5)$ & $-0.0015(5)$ \\
C4 & $0.0148(5)$ & $0.0174(5)$ & $0.0218(6)$ & $-0.0025(4)$ & $-0.0032(4)$ & $-0.0007(4)$ \\
C4a & $0.0153(5)$ & $0.0118(5)$ & $0.0171(5)$ & $-0.0006(4)$ & $-0.0038(4)$ & $-0.0007(4)$ \\
C5 & $0.0265(6)$ & $0.0168(5)$ & $0.0146(5)$ & $-0.0013(5)$ & $-0.0018(5)$ & $-0.0050(4)$ \\
C6 & $0.0351(7)$ & $0.0191(6)$ & $0.0170(6)$ & $-0.0030(5)$ & $-0.0085(5)$ & $-0.0076(5)$ \\
C7 & $0.0264(6)$ & $0.0193(6)$ & $0.0241(6)$ & $-0.0051(5)$ & $-0.0101(5)$ & $-0.0063(5)$ \\
C8 & $0.0184(6)$ & $0.0168(5)$ & $0.0188(6)$ & $-0.0025(4)$ & $-0.0038(4)$ & $-0.0057(4)$ \\
C8a & $0.0173(5)$ & $0.0116(5)$ & $0.0149(5)$ & $-0.0007(4)$ & $-0.0040(4)$ & $-0.0030(4)$ \\
C9 & $0.0158(5)$ & $0.0112(5)$ & $0.0141(5)$ & $-0.0009(4)$ & $-0.0030(4)$ & $-0.0018(4)$ \\
C9a & $0.0159(5)$ & $0.0109(5)$ & $0.0155(5)$ & $-0.0004(4)$ & $-0.0042(4)$ & $-0.0018(4)$ \\
C10 & $0.0166(5)$ & $0.0153(5)$ & $0.0146(5)$ & $-0.0002(4)$ & $0.0004(4)$ & $-0.0021(4)$ \\
C10a & $0.0201(6)$ & $0.0122(5)$ & $0.0136(5)$ & $0.0000(4)$ & $-0.0031(4)$ & $-0.0025(4)$ \\
C11 & $0.0146(5)$ & $0.0180(5)$ & $0.0155(5)$ & $-0.0037(4)$ & $-0.0041(4)$ & $-0.0076(4)$ \\
C12 & $0.0193(6)$ & $0.0200(6)$ & $0.0154(5)$ & $-0.0071(4)$ & $-0.0017(4)$ & $-0.0068(4)$ \\
C13 & $0.0299(6)$ & $0.0190(6)$ & $0.0189(6)$ & $-0.0054(5)$ & $-0.0016(5)$ & $-0.0037(5)$ \\
C14 & $0.0249(6)$ & $0.0269(6)$ & $0.0215(6)$ & $-0.0077(5)$ & $0.0039(5)$ & $-0.0070(5)$ \\
& & & & & & \\
& & & & & &
\end{tabular}

Geometric parameters $\left(\AA,{ }^{\circ}\right)$

\begin{tabular}{llll}
\hline $\mathrm{O}-\mathrm{C} 11$ & $1.2176(13)$ & $\mathrm{C} 9-\mathrm{C} 8 \mathrm{a}$ & $1.4024(15)$ \\
$\mathrm{C} 1-\mathrm{C} 2$ & $1.3603(17)$ & $\mathrm{C} 9-\mathrm{C} 9 \mathrm{a}$ & $1.4040(16)$ \\
$\mathrm{C} 1-\mathrm{H} 1$ & 0.9500 & $\mathrm{C} 9-\mathrm{C} 11$ & $1.5117(14)$ \\
$\mathrm{C} 2-\mathrm{C} 3$ & $1.4201(17)$ & $\mathrm{C} 9 \mathrm{a}-\mathrm{C} 1$ & $1.4300(16)$ \\
$\mathrm{C} 2-\mathrm{H} 2$ & 0.9500 & $\mathrm{C} 9 \mathrm{a}-\mathrm{C} 4 \mathrm{a}$ & $1.4365(15)$ \\
$\mathrm{C} 3-\mathrm{C} 4$ & $1.3615(17)$ & $\mathrm{C} 10-\mathrm{C} 10 \mathrm{a}$ & $1.3920(17)$ \\
$\mathrm{C} 3-\mathrm{H} 3$ & 0.9500 & $\mathrm{C} 10-\mathrm{H} 10$ & 0.9500 \\
$\mathrm{C} 4-\mathrm{C} 4 \mathrm{a}$ & $1.4297(16)$ & $\mathrm{C} 10 \mathrm{a}-\mathrm{C} 5$ & $1.4307(16)$ \\
$\mathrm{C} 4-\mathrm{H} 4$ & 0.9500 & $\mathrm{C} 10 \mathrm{a}-\mathrm{C} 8 \mathrm{a}$ & $1.4363(15)$ \\
$\mathrm{C} 4 \mathrm{a}-\mathrm{C} 10$ & $1.3948(16)$ & $\mathrm{C} 11-\mathrm{C} 12$ & $1.4864(15)$ \\
$\mathrm{C} 5-\mathrm{C} 6$ & $1.3579(18)$ & $\mathrm{C} 12-\mathrm{C} 13$ & $1.3277(17)$ \\
$\mathrm{C} 5-\mathrm{H} 5$ & 0.9500 & $\mathrm{C} 12-\mathrm{C} 14$ & $1.5020(16)$ \\
$\mathrm{C} 6-\mathrm{C} 7$ & $1.4207(17)$ & $\mathrm{C} 13-\mathrm{H} 13 \mathrm{~A}$ & 0.9500 \\
$\mathrm{C} 6-\mathrm{H} 6$ & 0.9500 & $\mathrm{C} 13-\mathrm{H} 13 \mathrm{~B}$ & 0.9500 \\
$\mathrm{C} 7-\mathrm{C} 8$ & $1.3618(16)$ & $\mathrm{C} 14-\mathrm{H} 14 \mathrm{~A}$ & 0.9800 \\
$\mathrm{C} 7-\mathrm{H} 7$ & 0.9500 & $\mathrm{C} 14-\mathrm{H} 14 \mathrm{~B}$ & 0.9800 \\
$\mathrm{C} 8-\mathrm{C} 8 \mathrm{a}$ & $1.4301(16)$ & $\mathrm{C} 14-\mathrm{H} 14 \mathrm{C}$ & 0.9800 \\
$\mathrm{C} 8-\mathrm{H} 8$ & 0.9500 & & $118.27(10)$ \\
$\mathrm{C} 1-\mathrm{C} 9 \mathrm{a}-\mathrm{C} 4 \mathrm{a}$ & & & $11.28(10)$ \\
$\mathrm{C} 2-\mathrm{C} 1-\mathrm{C} 9 \mathrm{a}$ & $118.42(10)$ & $\mathrm{C} 9-\mathrm{C} 8 \mathrm{a}-\mathrm{C} 10 \mathrm{a}$ & $1196(10)$ \\
$\mathrm{C} 2-\mathrm{C} 1-\mathrm{H} 1$ & $120.88(11)$ & $\mathrm{C} 8-\mathrm{C} 8 \mathrm{a}-\mathrm{C} 10 \mathrm{a}$ & $\mathrm{C} 8 \mathrm{C}-\mathrm{C} 9-\mathrm{C} 9 \mathrm{a}$ \\
$\mathrm{C} 9 \mathrm{a}-\mathrm{C} 1-\mathrm{H} 1$ & 119.6 & $\mathrm{C} 8-\mathrm{C} 9-\mathrm{C} 11$ &
\end{tabular}




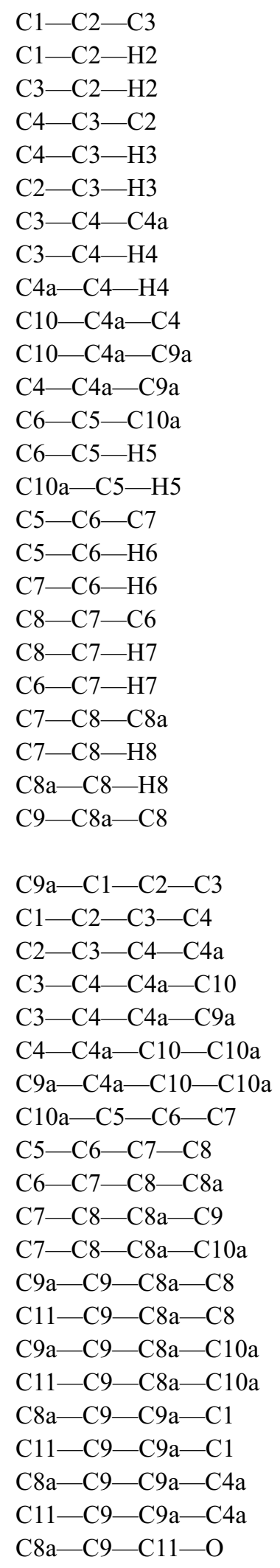

120.84 (11)

119.6

119.6

120.12 (11)

119.9

119.9

121.14 (11)

119.4

119.4

$121.93(10)$

$119.49(10)$

$118.57(10)$

$121.02(11)$

119.5

119.5

120.35 (11)

119.8

119.8

120.66 (11)

119.7

119.7

120.98 (11)

119.5

119.5

$122.61(10)$

$-0.39(17)$

$1.10(17)$

$-0.25(17)$

$-179.81(10)$

$-1.24(16)$

$179.63(10)$

$1.08(16)$

$-0.34(18)$

$0.28(18)$

$0.20(18)$

$179.79(10)$

$-0.58(16)$

$-178.68(9)$

$6.78(16)$

$1.70(16)$

$-172.84(9)$

$178.13(9)$

$-7.29(16)$

$-2.38(16)$

$172.20(9)$

88.94 (13)
$\mathrm{C} 9 \mathrm{a}-\mathrm{C} 9-\mathrm{C} 11$

$\mathrm{C} 9-\mathrm{C} 9 \mathrm{a}-\mathrm{C} 1$

$\mathrm{C} 9-\mathrm{C} 9 \mathrm{a}-\mathrm{C} 4 \mathrm{a}$

$\mathrm{C} 10 \mathrm{a}-\mathrm{C} 10-\mathrm{C} 4 \mathrm{a}$

$\mathrm{C} 10 \mathrm{a}-\mathrm{C} 10-\mathrm{H} 10$

$\mathrm{C} 4 \mathrm{a}-\mathrm{C} 10-\mathrm{H} 10$

$\mathrm{C} 10-\mathrm{C} 10 \mathrm{a}-\mathrm{C} 5$

$\mathrm{C} 10-\mathrm{C} 10 \mathrm{a}-\mathrm{C} 8 \mathrm{a}$

$\mathrm{C} 5-\mathrm{C} 10 \mathrm{a}-\mathrm{C} 8 \mathrm{a}$

$\mathrm{O}-\mathrm{C} 11-\mathrm{C} 12$

$\mathrm{O}-\mathrm{C} 11-\mathrm{C} 9$

$\mathrm{C} 12-\mathrm{C} 11-\mathrm{C} 9$

$\mathrm{C} 13-\mathrm{C} 12-\mathrm{C} 11$

$\mathrm{C} 13-\mathrm{C} 12-\mathrm{C} 14$

$\mathrm{C} 11-\mathrm{C} 12-\mathrm{C} 14$

C12-C13-H13A

C12-C13-H13B

H13A-C13-H13B

C12-C14-H14A

C12-C14-H14B

H14A-C14-H14B

C12-C14-H14C

$\mathrm{H} 14 \mathrm{~A}-\mathrm{C} 14-\mathrm{H} 14 \mathrm{C}$

H14B - C14-H14C

$\mathrm{C} 9 \mathrm{a}-\mathrm{C} 9-\mathrm{C} 11-\mathrm{O}$

$\mathrm{C} 8 \mathrm{a}-\mathrm{C} 9-\mathrm{C} 11-\mathrm{C} 12$

$\mathrm{C} 9 \mathrm{a}-\mathrm{C} 9-\mathrm{C} 11-\mathrm{C} 12$

$\mathrm{C} 9-\mathrm{C} 9 \mathrm{a}-\mathrm{C} 1-\mathrm{C} 2$

$\mathrm{C} 4 \mathrm{a}-\mathrm{C} 9 \mathrm{a}-\mathrm{C} 1-\mathrm{C} 2$

$\mathrm{C} 9-\mathrm{C} 9 \mathrm{a}-\mathrm{C} 4 \mathrm{a}-\mathrm{C} 10$

$\mathrm{C} 1-\mathrm{C} 9 \mathrm{a}-\mathrm{C} 4 \mathrm{a}-\mathrm{C} 10$

$\mathrm{C} 9-\mathrm{C} 9 \mathrm{a}-\mathrm{C} 4 \mathrm{a}-\mathrm{C} 4$

$\mathrm{C} 1-\mathrm{C} 9 \mathrm{a}-\mathrm{C} 4 \mathrm{a}-\mathrm{C} 4$

$\mathrm{C} 4 \mathrm{a}-\mathrm{C} 10-\mathrm{C} 10 \mathrm{a}-\mathrm{C} 5$

$\mathrm{C} 4 \mathrm{a}-\mathrm{C} 10-\mathrm{C} 10 \mathrm{a}-\mathrm{C} 8 \mathrm{a}$

$\mathrm{C} 10-\mathrm{C} 10 \mathrm{a}-\mathrm{C} 5-\mathrm{C} 6$

$\mathrm{C} 8 \mathrm{a}-\mathrm{C} 10 \mathrm{a}-\mathrm{C} 5-\mathrm{C} 6$

$\mathrm{C} 10-\mathrm{C} 10 \mathrm{a}-\mathrm{C} 8 \mathrm{a}-\mathrm{C} 9$

$\mathrm{C} 5-\mathrm{C} 10 \mathrm{a}-\mathrm{C} 8 \mathrm{a}-\mathrm{C} 9$

$\mathrm{C} 10-\mathrm{C} 10 \mathrm{a}-\mathrm{C} 8 \mathrm{a}-\mathrm{C} 8$

$\mathrm{C} 5-\mathrm{C} 10 \mathrm{a}-\mathrm{C} 8 \mathrm{a}-\mathrm{C} 8$

$\mathrm{O}-\mathrm{C} 11-\mathrm{C} 12-\mathrm{C} 13$

$\mathrm{C} 9-\mathrm{C} 11-\mathrm{C} 12-\mathrm{C} 13$

$\mathrm{O}-\mathrm{C} 11-\mathrm{C} 12-\mathrm{C} 14$

C9- $111-\mathrm{C} 12-\mathrm{C} 14$
$118.84(10)$

$122.57(10)$

$119.02(10)$

121.54 (10)

119.2

119.2

$121.77(10)$

$119.51(10)$

$118.72(11)$

$120.54(10)$

$119.34(10)$

$120.11(9)$

$120.05(10)$

$124.29(11)$

$115.66(10)$

120.0

120.0

120.0

109.5

109.5

109.5

109.5

109.5

109.5

$-85.73(13)$

$-91.47(12)$

$93.86(12)$

$178.37(10)$

$-1.12(16)$

$0.99(15)$

$-179.50(10)$

$-177.61(9)$

$1.90(15)$

$178.48(10)$

$-1.78(16)$

$179.69(10)$

-0.05 (17)

0.39 (16)

-179.85 (9)

$-179.25(10)$

$0.51(15)$

179.37 (11)

$-0.21(16)$

$0.10(15)$

$-179.48(10)$ 
Hydrogen-bond geometry $\left(\AA,{ }^{\circ}\right)$

\begin{tabular}{lllll}
\hline$D-\mathrm{H} \cdots A$ & $D-\mathrm{H}$ & $\mathrm{H} \cdots A$ & $D \cdots A$ & $D-\mathrm{H} \cdots A$ \\
\hline $\mathrm{C} 3-\mathrm{H} 3 \cdots \mathrm{O}^{\mathrm{i}}$ & 0.95 & 2.48 & $3.3747(16)$ & 157 \\
\hline
\end{tabular}

Symmetry code: (i) $x-1, y, z$.

Geometric parameters $\left(\AA,{ }^{\circ}\right)$

Calculated values using RM06-2X/6-31+G(d) for optimized isolated molecule given in square brackets

\begin{tabular}{|c|c|c|}
\hline $\mathrm{O}-\mathrm{C} 11$ & $1.2176(13)$ & [1.217] \\
\hline $\mathrm{C} 1-\mathrm{C} 2$ & $1.3603(17)$ & [1.365] \\
\hline $\mathrm{C} 1-\mathrm{H} 1$ & 0.9500 & [1.086] \\
\hline $\mathrm{C} 2-\mathrm{C} 3$ & $1.4201(17)$ & [1.428] \\
\hline $\mathrm{C} 2-\mathrm{H} 2$ & 0.9500 & [1.086] \\
\hline $\mathrm{C} 3-\mathrm{C} 4$ & $1.3615(17)$ & [1.364] \\
\hline $\mathrm{C} 3-\mathrm{H} 3$ & 0.9500 & {$[1.086]$} \\
\hline $\mathrm{C} 4-\mathrm{C} 4 \mathrm{a}$ & $1.4297(16)$ & [1.432] \\
\hline $\mathrm{C} 4-\mathrm{H} 4$ & 0.9500 & [1.087] \\
\hline $\mathrm{C} 4 \mathrm{a}-\mathrm{C} 10$ & $1.3948(16)$ & [1.396] \\
\hline $\mathrm{C} 5-\mathrm{C} 6$ & $1.3579(18)$ & {$[1.364]$} \\
\hline $\mathrm{C} 5-\mathrm{H} 5$ & 0.9500 & [1.087] \\
\hline $\mathrm{C} 6-\mathrm{C} 7$ & $1.4207(17)$ & [1.428] \\
\hline $\mathrm{C} 6-\mathrm{H} 6$ & 0.9500 & [1.086] \\
\hline $\mathrm{C} 7-\mathrm{C} 8$ & $1.3618(16)$ & [1.365] \\
\hline $\mathrm{C} 7-\mathrm{H} 7$ & 0.9500 & {$[1.086]$} \\
\hline $\mathrm{C} 8-\mathrm{C} 8 \mathrm{a}$ & $1.4301(16)$ & [1.434] \\
\hline $\mathrm{C} 8-\mathrm{H} 8$ & 0.9500 & [1.087] \\
\hline $\mathrm{C} 9-\mathrm{C} 8 \mathrm{a}$ & $1.4024(15)$ & [1.404] \\
\hline $\mathrm{C} 9-\mathrm{C} 9 \mathrm{a}$ & $1.4040(16)$ & [1.404] \\
\hline C9- $-\mathrm{C} 11$ & $1.5117(14)$ & [1.509] \\
\hline $\mathrm{C} 9 \mathrm{a}-\mathrm{C} 1$ & $1.4300(16)$ & [1.434] \\
\hline $\mathrm{C} 9 \mathrm{a}-\mathrm{C} 4 \mathrm{a}$ & $1.4365(15)$ & [1.437] \\
\hline $\mathrm{C} 10-\mathrm{C} 10 \mathrm{a}$ & $1.3920(17)$ & [1.396] \\
\hline $\mathrm{C} 10-\mathrm{H} 10$ & 0.9500 & [1.089] \\
\hline $\mathrm{C} 10 \mathrm{a}-\mathrm{C} 5$ & $1.4307(16)$ & [1.432] \\
\hline $\mathrm{C} 10 \mathrm{a}-\mathrm{C} 8 \mathrm{a}$ & $1.4363(15)$ & [1.436] \\
\hline $\mathrm{C} 11-\mathrm{C} 12$ & $1.4864(15)$ & {$[1.497]$} \\
\hline $\mathrm{C} 12-\mathrm{C} 13$ & $1.3277(17)$ & [1.339] \\
\hline $\mathrm{C} 12-\mathrm{C} 14$ & $1.5020(16)$ & [1.502] \\
\hline $\mathrm{C} 13-\mathrm{H} 13 \mathrm{~A}$ & 0.9500 & [1.087] \\
\hline C13-H13B & 0.9500 & {$[1.087]$} \\
\hline $\mathrm{C} 14-\mathrm{H} 14 \mathrm{~A}$ & 0.9500 & [1.093] \\
\hline C14-H14B & 0.9500 & [1.095] \\
\hline $\mathrm{C} 14-\mathrm{H} 14 \mathrm{C}$ & 0.9500 & [1.095] \\
\hline $\mathrm{C} 2-\mathrm{C} 1-\mathrm{C} 9 \mathrm{a}$ & $120.88(11)$ & [120.8] \\
\hline $\mathrm{C} 2-\mathrm{C} 1-\mathrm{H} 1$ & 119.6 & [120.0] \\
\hline $\mathrm{C} 9 \mathrm{a}-\mathrm{C} 1-\mathrm{H} 1$ & 119.6 & [119.1] \\
\hline
\end{tabular}




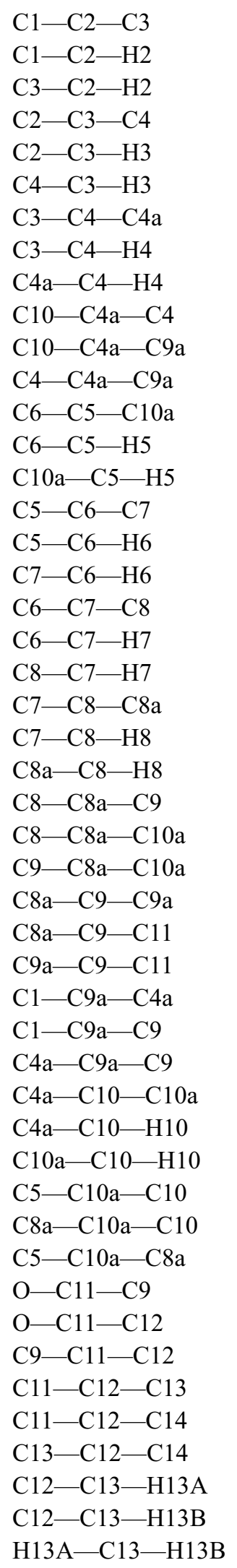

120.84 (11)

119.6

119.6

120.12 (11)

119.9

119.9

121.14 (11)

119.4

119.4

$121.93(10)$

$119.49(10)$

$118.57(10)$

$121.02(11)$

119.5

119.5

120.35 (11)

119.8

119.8

120.66 (11)

119.7

119.7

120.98 (11)

119.5

119.5

$122.61(10)$

$118.27(10)$

$119.11(10)$

$121.28(10)$

$119.66(10)$

$118.84(10)$

$118.42(10)$

$122.57(10)$

$119.02(10)$

$121.54(10)$

119.2

119.2

121.77 (10)

$119.51(10)$

118.72 (11)

$119.34(10)$

120.54 (10)

120.11 (9)

120.05 (10)

$115.66(10)$

$124.29(11)$

120.0

120.0

120.0
[120.8]

[119.9]

[119.4]

[120.2]

[119.5]

[120.3]

[120.9]

[120.8]

[118.3]

[121.6]

[119.5]

[119.0]

[120.9]

[120.8]

[118.3]

[120.2]

[120.3]

[119.5]

[120.7]

[119.4]

[119.9]

[120.9]

[119.9]

[119.2]

[122.5]

[118.3]

[119.2]

[121.2]

[119.7]

[119.0]

[118.4]

[122.5]

[119.1]

[121.5]

[119.2]

[119.2]

[121.6]

[119.4]

[119.0]

[120.5]

[120.2]

[119.3]

[120.4]

[115.4]

[124.2]

[121.8]

[121.0]

[117.2] 
C12-C14-H14A

C12-C14-H14B

$\mathrm{C} 12-\mathrm{C} 14-\mathrm{H} 14 \mathrm{C}$

$\mathrm{H} 14 \mathrm{~A}-\mathrm{C} 14-\mathrm{H} 14 \mathrm{~B}$

$\mathrm{H} 14 \mathrm{~A}-\mathrm{C} 14-\mathrm{H} 14 \mathrm{C}$

$\mathrm{H} 14 \mathrm{~B}-\mathrm{C} 14-\mathrm{H} 14 \mathrm{C}$

$\mathrm{C} 9 \mathrm{a}-\mathrm{C} 1-\mathrm{C} 2-\mathrm{C} 3$

$\mathrm{C} 1-\mathrm{C} 2-\mathrm{C} 3-\mathrm{C} 4$

$\mathrm{C} 2-\mathrm{C} 3-\mathrm{C} 4-\mathrm{C} 4 \mathrm{a}$

$\mathrm{C} 3-\mathrm{C} 4-\mathrm{C} 4 \mathrm{a}-\mathrm{C} 10$

$\mathrm{C} 3-\mathrm{C} 4-\mathrm{C} 4 \mathrm{a}-\mathrm{C} 9 \mathrm{a}$

$\mathrm{C} 4-\mathrm{C} 4 \mathrm{a}-\mathrm{C} 10-\mathrm{C} 10 \mathrm{a}$

$\mathrm{C} 9 \mathrm{a}-\mathrm{C} 4 \mathrm{a}-\mathrm{C} 10-\mathrm{C} 10 \mathrm{a}$

$\mathrm{C} 10 \mathrm{a}-\mathrm{C} 5-\mathrm{C} 6-\mathrm{C} 7$

$\mathrm{C} 5-\mathrm{C} 6-\mathrm{C} 7-\mathrm{C} 8$

$\mathrm{C} 6-\mathrm{C} 7-\mathrm{C} 8-\mathrm{C} 8 \mathrm{a}$

$\mathrm{C} 7-\mathrm{C} 8-\mathrm{C} 8 \mathrm{a}-\mathrm{C} 9$

$\mathrm{C} 7-\mathrm{C} 8-\mathrm{C} 8 \mathrm{a}-\mathrm{C} 10 \mathrm{a}$

$\mathrm{C} 9 \mathrm{a}-\mathrm{C} 9-\mathrm{C} 8 \mathrm{a}-\mathrm{C} 8$

$\mathrm{C} 11-\mathrm{C} 9-\mathrm{C} 8 \mathrm{a}-\mathrm{C} 8$

$\mathrm{C} 9 \mathrm{a}-\mathrm{C} 9-\mathrm{C} 8 \mathrm{a}-\mathrm{C} 10 \mathrm{a}$

$\mathrm{C} 11-\mathrm{C} 9-\mathrm{C} 8 \mathrm{a}-\mathrm{C} 10 \mathrm{a}$

$\mathrm{C} 8 \mathrm{a}-\mathrm{C} 9-\mathrm{C} 9 \mathrm{a}-\mathrm{C} 1$

$\mathrm{C} 11-\mathrm{C} 9-\mathrm{C} 9 \mathrm{a}-\mathrm{C} 1$

$\mathrm{C} 8 \mathrm{a}-\mathrm{C} 9-\mathrm{C} 9 \mathrm{a}-\mathrm{C} 4 \mathrm{a}$

$\mathrm{C} 11-\mathrm{C} 9-\mathrm{C} 9 \mathrm{a}-\mathrm{C} 4 \mathrm{a}$

$\mathrm{C} 8 \mathrm{a}-\mathrm{C} 9-\mathrm{C} 11-\mathrm{O}$

$\mathrm{C} 9 \mathrm{a}-\mathrm{C} 9-\mathrm{C} 11-\mathrm{O}$

$\mathrm{C} 8 \mathrm{a}-\mathrm{C} 9-\mathrm{C} 11-\mathrm{C} 12$

$\mathrm{C} 9 \mathrm{a}-\mathrm{C} 9-\mathrm{C} 11-\mathrm{C} 12$

$\mathrm{C} 9-\mathrm{C} 9 \mathrm{a}-\mathrm{C} 1-\mathrm{C} 2$

$\mathrm{C} 4 \mathrm{a}-\mathrm{C} 9 \mathrm{a}-\mathrm{C} 1-\mathrm{C} 2$

$\mathrm{C} 9-\mathrm{C} 9 \mathrm{a}-\mathrm{C} 4 \mathrm{a}-\mathrm{C} 10$

$\mathrm{C} 1-\mathrm{C} 9 \mathrm{a}-\mathrm{C} 4 \mathrm{a}-\mathrm{C} 10$

$\mathrm{C} 9-\mathrm{C} 9 \mathrm{a}-\mathrm{C} 4 \mathrm{a}-\mathrm{C} 4$

$\mathrm{C} 1-\mathrm{C} 9 \mathrm{a}-\mathrm{C} 4 \mathrm{a}-\mathrm{C} 4$

$\mathrm{C} 4 \mathrm{a}-\mathrm{C} 10-\mathrm{C} 10 \mathrm{a}-\mathrm{C} 5$

$\mathrm{C} 4 \mathrm{a}-\mathrm{C} 10-\mathrm{C} 10 \mathrm{a}-\mathrm{C} 8 \mathrm{a}$

$\mathrm{C} 10-\mathrm{C} 10 \mathrm{a}-\mathrm{C} 5-\mathrm{C} 6$

$\mathrm{C} 8 \mathrm{a}-\mathrm{C} 10 \mathrm{a}-\mathrm{C} 5-\mathrm{C} 6$

$\mathrm{C} 10-\mathrm{C} 10 \mathrm{a}-\mathrm{C} 8 \mathrm{a}-\mathrm{C} 9$

$\mathrm{C} 5-\mathrm{C} 10 \mathrm{a}-\mathrm{C} 8 \mathrm{a}-\mathrm{C} 9$

$\mathrm{C} 10-\mathrm{C} 10 \mathrm{a}-\mathrm{C} 8 \mathrm{a}-\mathrm{C} 8$

$\mathrm{C} 5-\mathrm{C} 10 \mathrm{a}-\mathrm{C} 8 \mathrm{a}-\mathrm{C} 8$

$\mathrm{O}-\mathrm{C} 11-\mathrm{C} 12-\mathrm{C} 13$

C9-C11-C12-C13

$\mathrm{O}-\mathrm{C} 11-\mathrm{C} 12-\mathrm{C} 14$
109.5

109.5

109.5

109.5

109.5

109.5

$-0.39(17)$

1.10 (17)

$-0.25(17)$

$-179.81(10)$

-1.24 (16)

179.63 (10)

1.08 (16)

-0.34 (18)

0.28 (18)

$0.20(18)$

179.79 (10)

-0.58 (16)

$-178.68(9)$

6.78 (16)

1.70 (16)

$-172.84(9)$

178.13 (9)

-7.29 (16)

-2.38 (16)

$172.20(9)$

88.94 (13)

$-85.73(13)$

$-91.47(12)$

93.86 (12)

178.37 (10)

-1.12 (16)

0.99 (15)

$-179.50(10)$

$-177.61(9)$

$1.90(15)$

$178.48(10)$

$-1.78(16)$

179.69 (10)

-0.05 (17)

0.39 (16)

$-179.85(9)$

$-179.25(10)$

$0.51(15)$

179.37 (11)

$-0.21(16)$

$0.10(15)$
[110.9]

[110.6]

[110.6]

[109.2]

[109.1]

[106.5]

$[-0.1]$

$[-0.1]$

[0.2]

[179.7]

$[-0.2]$

[-179.9]

$[0.0]$

$[-0.2]$

[0.3]

[0.0]

[-179.9]

$[-0.3]$

[179.0]

[0.2]

$[-0.6]$

[-179.4]

[-179.8]

[-1.1]

[1.1]

[179.8]

[102.3]

$[-76.5]$

[-78.8]

[102.4]

[-179.0]

[0.1]

$[-0.7]$

[-179.9]

[179.1]

[0.0]

[-179.7]

[0.4]

[180.0]

[-0.1]

[-0.1]

[180.0]

[-179.7]

[0.4]

[172.7]

[-6.1]

[-5.8] 
